\title{
CORRESPONDENCE
}

\section{DNA X-ray data}

SIR - We have recently suggested ${ }^{1}$ that the available X-ray diffraction data from B DNA are consistent with a model for the structure of DNA which we have called the "side-by-side" model and in which DNA strands are linked together in pairs of the Watson-Crick type but are not wound into a continuous right-handed helix. Our model has however been alluded to 2.3 in terms that suggest that alternatives to the double-helix model have been finally disposed of. We wish now to emphasize that existing X-ray data on B DNA are not in themselves sufficient for an unambiguous decision for or against either model.

Naturally occurring specimens of B DNA do not form single crystals of good quality, and the resolution of available $\mathrm{X}$-ray data is very limited. For these reasons, and because there is rotational disorder in all specimens of B DNA so far reported, the only standard crystallographic quantity that can be immediatcly calculated from the $\mathrm{X}$-ray data is some cylindrical average of the Patterson function, which must in practice be compared with the corresponding function calculated from molecular models.

We have shown ${ }^{1}$ that in the analysis of B DNA data, there are advantages in using what we call the axial Patterson function (essentially the cylindrically averaged Patterson function with the radial coordinate zero). Briefly, the axial Patterson function is less sensitive than the Patterson function itself to imperfections of diffraction data. Of necessity, the axial Patterson function, which averages out much of the fine detail inherent in the observed diffraction patterns, can only be used for preliminary tests of the agreement between model structures and the X-ray data.

The nub of our argument is that the axial Pattcrson function computed from the data agrees (within the apparent experimental error) with that calculated from the side-byside model but not with that computed from the double-helix model refined by Arnott and Hukins ${ }^{4}$. We do not of course claim that the doublc-hclix model can therefore be dismissed - the quality of the data is so poor. But in the circumstances one wonders about the value of sophisticated refinements of the double-helix model (for example, ref. 4).

We are also surprised at the recent claim by Greenall et al. ${ }^{5}$ that the double-helix model is in much better agreement than the side-by-side model with the diffraction patterns measured from paracrystalline B DNA specimens. We find roughly equal disagreement for both models. (We do not wish to make too much of what we feel is an anomaly of the double helix on the tenth layer line, because it could well be an artefact of excessive refinement based on inadequate data.)

For such reasons, it must be concluded that available X-ray data are at best an equivocal means of deciding between alternative DNA models. So much is acknowledged by Crick et $a l .{ }^{6}$. For X-ray analysis to provide unambiguous distinction between different models of DNA, it will be necessary for much better crystals to be prepared from well characterized polynucleotides. In the meantime, while no doubt the merits of alternative DNA models will be assessed by considerations other than those deriving from
$\mathrm{X}$-ray data, it is prudent that the present insufficiency of the latter should be widely and openly acknowledged.

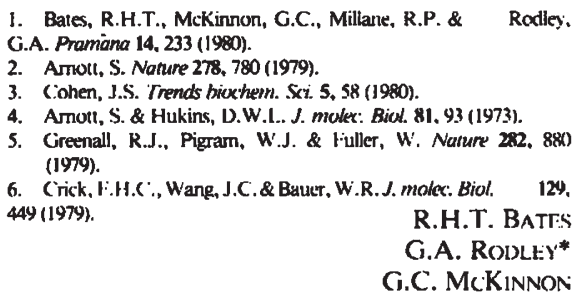

Electrical Engineering and *Chemistry

Departments University of Canterbury, Christchurch New Zealand

\section{Andrei Sakharov}

SIR - The Olympic games are over; the hopes that Academician Andrei Sakharov might be allowed to leave his internal exile in Gorky and return to Moscow are over now too. Professor Sakharov has been exiled for over nine months and has no contact with his scientific colleagues, is not allowed to take part in scminars or lectures, has no access to scientific information so crucial for his research. (Books and journals have to be brought to him by his relatives from Moscow, which usually takes weeks or months. Since January, his colleagues from Lebedev Institute have been allowed to visit him only three times!)

In spite of this, he has managed to write three scientific papers in Gorky, and English trarislations have been published at SLAC, Stanford University (Estimate of the QuarkGluon Coupling Constant; Cosmological Models of the University with Rotation of Time's Arrow; Mass Formula for Mesons and Baryons)

I am convinced that it is time now for the world scientific community to escalate the efforts to help our distinguished Soviet colleague. From Andrei Sakharov's last communication it is obvious that he is mainly missing the information about what is going on in physics.

It should be very easy for the physicists to break down this information barrier. Let the theoretical institutes, laboratories and groups from all over the world begin sending to Professor Sakharov their preprints, lecture notes, reports. Send them by registered mail with the red "Avis de Reception" card to:

Professor Andrei Sakharov

Prospekt Gagarina 214, kv.3

Scherbinka 2, Gorky, USSR

Don't hesitate to ask your local post of fice to investigate if the red card is not returned to you with Andrei Sakharov's signature within about a month. Your local post office is obliged, according to international postal convention, to make the investigation and, if unable to provide proof that the mai! was delivered, to pay you compensation.

There is no doubt that such world-wide action will not only provide Professor Sakharov with necessary scientific information but will also represent considerable moral support for him. It will be important for Soviet authorities too: they will see that Andrei Sakharov's case is not at all forgotten by the world scientific community'

Research Institute for Physics,

F. JANOUCH

Stockholm, Sweden

\section{Preventing plagiarism}

SIR - The article "An outbreak of piracy in the literature"' (Nature 12 June, p.429) shocked me deeply. In December 1978 Dr Alsabti scnt manuscripts to us also, with the headed paper of the M.D. Anderson Tumor Institute. Again, he gave a private address in Houston for correspondence and made acknowledgement to the Jordan Royal Family. Fortunately for us, he grossly overdid it, sending five manuscripts within a month. A call to M.D. Anderson helped us in taking the decision to reject the five manuscripts.

An old custom in the Italian universities called for the approving signature of the professor on the front page of a manuscript. Although I do not agree with a system which may impair scientific freedom, the present incident should teach us how important it is to ascertain directly from the host institution the credentials of an intended author, particularly when complcx international relationships are apparent.

UMBERTOVERONES

Istituto Nazionale per lo Studio

e la Cura dei Tumori

Milan, Italy

\section{Pesticides as poisons}

SIR- Peter Clarke (Nature 18 September p.184) defines a poison, but fails to note that "the dose alone determines the poison" (Paracelsus, 1564), a point that was emphasized correctly in Nature 28 August p.832. He says high blood levels of DDT in Guatemala were "the corollary" of an "aggressive marketing campaign" by a transnational corporation, and that cigarettes and pesticides are "harmful products". Cigarettes are harmful to human beings, pesticides to pests. The use of DDT in Guatemala was started by the World Heaith Organization and the United Nations International Children's Emergency Fund to control endemic malaria in 1,500 Guatemalan communities. A reduction in malaria was achieved.

THOMAS H. JUKES

Department of Biophysics and

Medical Physics,

University of California,

Berkeley, California

SIR - Of necessity, I have had to become something of an "applied toxicologist".

The only still-unfalsified definition of a poison is that of Paracelsus "Dosis facit venenum"; the definition of fered by your correspondent Peler Clarke (Nature 18 September, p. 184) contains less of the essential nature of a poison, and therefore fails. Nature, conscquently, has avoided polemic (unlike your correspondent), and has indeed abandoned prejudice. Dr Clarke's letter is a brilliant piece of special pleading on behalf of a political stance - and I congratulate Nature, Sir, on your stand for true objectivity.

l an about to retire from full-time employment as a scientist, but 1 hope to maintain an attitude of 'informed scepticism'.

Rodent Research Laboratory

Tolworth Lahoratory, Surrey, UK 